\title{
A wearable cardioverter defibrillator for prevention of sudden cardiac death in a hematologic patient-a case report
}

\author{
Stefan Hatzl · Hildegard T. Greinix · Philipp Kreuzer · Eduard Schulz • Peter Neumeister · Daniel Scherr
}

Received: 26 June 2019 / Accepted: 16 July 2019 / Published online: 7 August 2019

(C) The Author(s) 2019

\begin{abstract}
Summary The wearable cardioverter defibrillator (WCD) is a suitable option to overcome life-threatening episodes of malignant arrhythmia. There is much experience in the use of these devices in patients with idiopathic arrhythmia as well as in patients at a high risk for sudden cardiac death. However, there is lack of experience with WCD usage in hematologic patients with a limited expected lifespan. Here, we report a rare case of malignant arrhythmia in a patient with cardiac relapse of diffuse large B-cell lymphoma (DLBCL) and the option of WCD use. WCD provides the possibility of bridging to implantation of a cardioverter defibrillator (ICD) in cases with a high risk of hemorrhage as well as being a definite therapy in patients with limited life expectance.
\end{abstract}

Keywords WCD · Lymphoma - Arrhythmia - Cardiac relapse $\cdot(I C D)$ implantable cardioverter-defibrillator

\section{Introduction}

The implantable cardioverter defibrillator (ICD) is a cornerstone in the prevention of sudden cardiac death in patients with cardiomyopathy as well as survivors of idiopathic ventricular arrhythmias. The indications for this device are strict and exclude pa-

\footnotetext{
S. Hatzl $(\bowtie) \cdot$ H. Greinix $\cdot$ E. Schulz $\cdot$ P. Neumeister

Division of Hematology, Department of Internal Medicine,

Medical University of Graz, Auenbruggerplatz 15, 8036 Graz, Austria

stefan.hatzl@medunigraz.at

P. Kreuzer

Division of Emergency Medicine, Department of Internal Medicine, Medical University of Graz, Graz, Austria

D. Scherr

Division of Cardiology, Department of Internal Medicine, Medical University of Graz, Graz, Austria
}

tients with a limited expected lifespan as well as patients with an expected improvement of cardiac function [1]. Cardiac involvement or sudden cardiac death within hematologic diseases is a rare finding. In a large autopsy study, $8 \%$ of patients deceased due to a lymphatic malignancy showed cardiac involvement [2]. There is a lack of published data of cardiac involvement within myeloid neoplasia. Only case reports on this topic are available $[3,4]$. The vast majority of cases can be effectively treated by initiation of a specific therapy, leading to a rapid improvement of cardiac function and preventing sudden cardiac death [5]. The wearable cardioverter defibrillator (WCD) is a well-established opportunity to bridge such patients [6].

In this case report, we describe the use of WCD in a patient with cardiac relapse of an aggressive lymphoma.

\section{Case}

A 61-year-old man was admitted to the emergency department with fatigue and bilateral leg edema. The patient had been diagnosed with a primary testicular diffuse large B cell lymphoma 1 year previously. He had undergone chemotherapy according to the R-CHOP 21 protocol and had additionally received three cycles of high-dose methotrexate for central nervous system (CNS) prophylaxis. First complete remission (CR) was achieved after four cycles of R-CHOP 21. Physical examination revealed wet breath sounds as well as massive bilateral leg edema. Laboratory findings showed high pro-BNP (19,500 [0-100 pg/ml]) and elevated high-sensitive troponin $\mathrm{T}(123[0-14 \mathrm{pg} / \mu \mathrm{l}])$. Cardiac ultrasound demonstrated pericardial effusion ( $3 \mathrm{~cm}$ end-diastolic) and paradox septum movement as well as solid formations swinging in the effusion. During ongoing examination, the patient became un- 


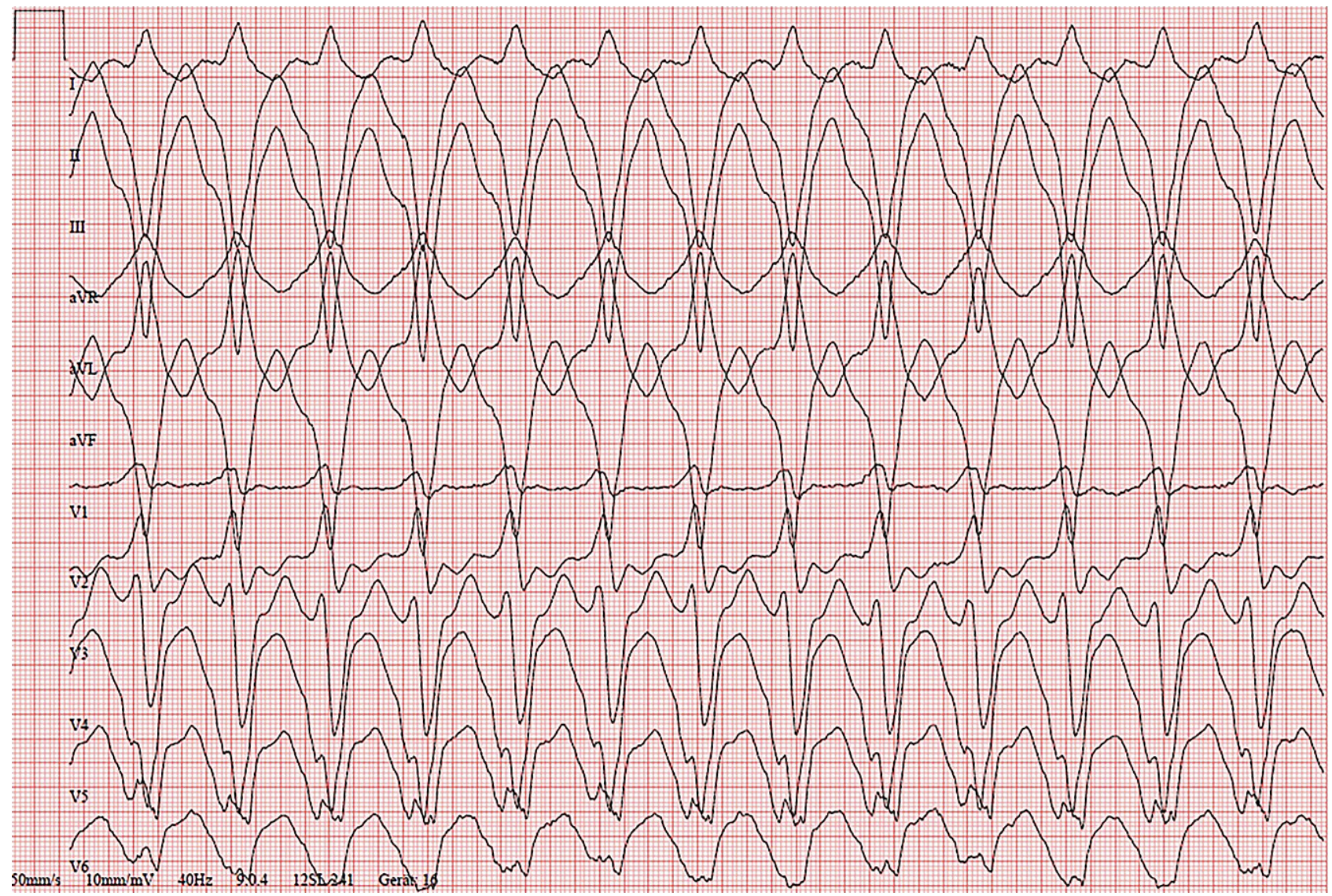

Fig. 1 Electrocardiography (12-channel) at time of cardiac arrest, showing sustained monomorphic ventricular tachycardia

Fig. 2 Magnetic resonance imaging showing Diffuse large B-cell lymphoma (DLBC) infiltration of the left ventricular myocardium with epifocal edema (red arrows)

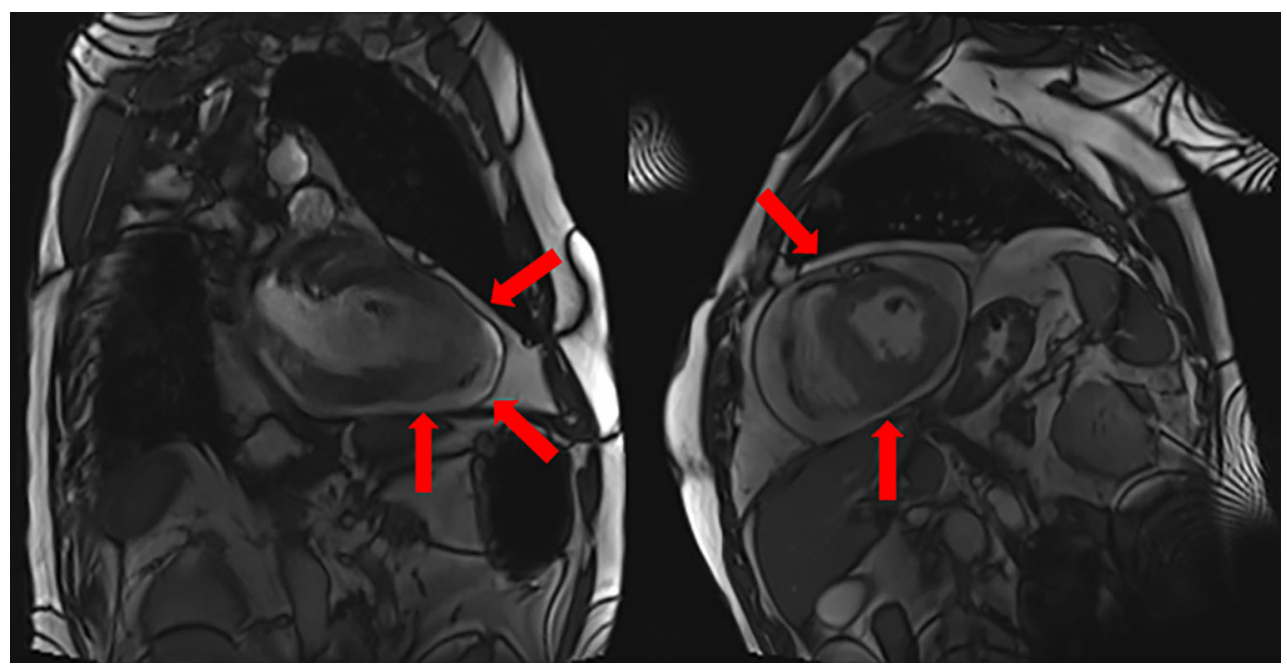

conscious and suffered cardiac arrest. Immediate cardiopulmonary reanimation was initiated. Ventricular fibrillation was documented by 12-channel electrocardiography (Fig. 1). After $10 \mathrm{~min}$ of resuscitation and defibrillation, the patient achieved return of spontaneous circulation (ROSC). After stabilization of his vital signs, cardiac MRI was performed, revealing myocardial infiltration of the left ventricle most likely due to lymphomatous formations (Fig. 2). Due to my- ocardial location and reduced patient performance score, biopsy could not be performed. Dexamethasone with a dose of $40 \mathrm{mg} /$ day was administered as the first step of lymphoma salvage treatment. The PET imaging confirmed the MRI finding of cardiac relapse, with high tracer uptake in the whole myocardium as well as involvement of mediastinal lymph nodes. For prevention of further life-threatening arrhythmias and sudden cardiac death, the patient was equipped with 
a WCD. Within the course of relapse, the patient developed acute renal failure. This fact disqualifies him from standard platinum-containing salvage therapy. Therefore, pixantrone combined with rituximab and dexamethasone was used, leading to disappearance of cardiac effusion and improvement of cardiac function as well as partial response of the DLBCL, with remaining 18-fludeoxyglucose uptake in mediastinal lymph nodes. After commencement of pixantrone plus rituximab, no further life-threatening arrhythmia were observed. Unfortunately, the patient developed a third, refractory mediastinal relapse of his lymphoma and died 10 weeks after diagnosis of his cardiac relapse.

\section{Conclusion}

In this report, we describe a very rare case of cardiac relapse of a DLBCL and the successful application of a WCD, thereby preventing further episodes of ventricular fibrillation and bridging the patient until initiation of salvage chemotherapy. WCD allows safe administration of anti-lymphoma treatment.

Funding Open access funding provided by Medical University of Graz.

Conflict of interest S. Hatzl, H. Greinix, P. Kreuzer, E. Schulz, P. Neumeister, and D. Scherr declare that they have no competing interests.

Open Access This article is distributed under the terms of the Creative Commons Attribution 4.0 International License (http://creativecommons.org/licenses/by/4.0/), which permits unrestricted use, distribution, and reproduction in any medium, provided you give appropriate credit to the original author(s) and the source, provide a link to the Creative Commons license, and indicate if changes were made.

\section{References}

1. Priori SG, Blomstrom-Lundqvist C, Mazzanti A, Blom N, Borggrefe M, Camm J, et al. 2015 ESC Guidelines for the management of patients with ventricular arrhythmias and the prevention of sudden cardiac death: The Task Force for the Management of Patients with Ventricular Arrhythmias and the Prevention of Sudden Cardiac Death of the European Society of Cardiology (ESC) Endorsed by: Association for European Paediatric and Congenital Cardiology (AEPC). Europace. 2015;17(11):1601-87.

2. McDonnell PJ, Mann RB, Bulkley BH. Involvement of the heart by malignant lymphoma: a clinicopathologic study. Cancer. 1982;49(5):944-51.

3. Kim TY, Jung JI, Kim YJ, Kim HW, Lee HG. CT and MRI evaluation of cardiac complications in patients with hematologic diseases: a pictorial review. IntJCardiovasc Imaging. 2015;31(Suppl2):159-67.

4. Tsai J, Lee EY. MDCT imaging findings of extramedullary granulocytic sarcoma of the heart. J Thorac Imaging. 2010;25(1):W14-W6.

5. Lyman GH, Barron RL, Natoli JL, Miller RM. Systematic review of efficacy of dose-dense versus non-dose-dense chemotherapy in breast cancer, non-Hodgkin lymphoma, and non-small cell lung cancer. Crit Rev Oncol Hematol. 2012;81(3):296-308.

6. Odeneg T, Ebner C, Mortl D, Keller H, Dirninger A, Stix G, et al. Indications for and outcome in patients with the wearable cardioverter-defibrillator in a nurse-based training programme: results of the Austrian WCD Registry. Eur J Cardiovasc Nurs. 2019;18(1):75-83.

Publisher's Note Springer Nature remains neutral with regard to jurisdictional claims in published maps and institutional affiliations.

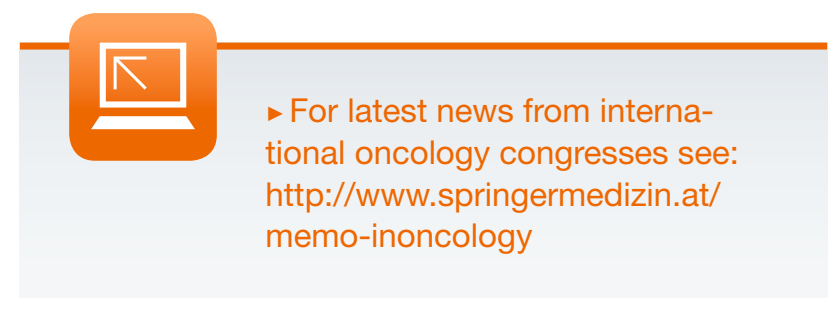

